\title{
Does the Perceptual Load Hypothesis Predict Auditory Distractibility?
}

\author{
Jacob Schlaegel (schlaegel.22@,osu.edu) \\ Department of Psychology, The Ohio State University at Newark \\ 1179 University Dr., Newark, OH 43056, USA \\ Christopher W. Robinson (robinson.777@osu.edu) \\ Department of Psychology, The Ohio State University at Newark \\ 1179 University Dr., Newark, OH 43056, USA
}

\begin{abstract}
Attention is a valuable resource with limited capacity, so knowing what will distract us during important tasks can be crucial in life. There is a lot of support for the Perceptual Load Hypothesis (PLH) when examining visual distractibility, however, less research has examined if PLH can predict auditory distractibility. Participants in the current study completed visual selective attention tasks while being presented with auditory and visual distractions under low/high perceptual loads. While visual distractors had a larger effect on the visual selective attention task, we increased auditory distractor effects by requiring participants to periodically respond to the auditory information. Our results showed no support for PLH with auditory distractors, and instead showed the opposite pattern, with auditory distractors having a larger effect under high perceptual load. These findings have important implications for our understanding of selective attention and shed light on tasks that require the processing of multisensory information.
\end{abstract}

Keywords: Selective Attention, Multisensory Processing, Perceptual Load

\section{Introduction}

In life, there are many situations that require us to focus our selective attention on a task and ignore irrelevant information. For example, working on homework in a noisy environment and driving in heavy downtown traffic both require focused attention and poor selective attention can result in being unproductive or contributing to a deadly accident. Thus, it is important to understand what factors account for increased distractibility. While there is considerable support for the Perceptual Load Hypothesis in the visual modality (Lavie, 1995; Lavie \& Tsal, 1994; see also Murphy, Groeger, \& Greene, 2016, for a review), less research has examined if the Perceptual Load Hypothesis can predict the effects of auditory or cross-modal distractors on selective attention. The current study contributes to this research by examining the filtering of auditory and visual distractors under low and high load conditions while participants are performing a visual selective attention task.

The Perceptual Load Hypothesis (PLH) predicts that visual distractors should be more distracting under low perceptual load (when there is less information to process) than under higher load. The underlying idea is that in low load conditions participants have more available attentional resources to detect the distractors, whereas, they are less likely to see/hear distractors under high load. For example, in
Lavie (1995), participants had to quickly respond to targets and ignore distractors. Distractors were either compatible (target and distractor were identical and associated with the same response), incompatible (target was associated with one response and distractor was associated with a different response), or neutral (distractors were not associated with any response). In Experiment 1, the high load condition had five distractor stimuli and one target and the low load condition had just the target and one distractor. Lavie (1995) found that incompatible distractors interfered more in the low load condition compared to the high load condition (see also Murphy, Groeger, \& Greene, 2016, for a review).

Studies that have looked into auditory/cross-modal distractions have produced mixed findings (Macdonald \& Lavie, 2011; Robinson, Hawthorn, Rahman, 2018; Tellinghusien \& Nowak 2003; see also Murphy et al. 2017 for a review). For example, Macdonald and Lavie (2011) manipulated the perceptual load in the visual modality and examined auditory detection. Consistent with PLH, participants were more likely to detect the auditory stimulus under low visual load, therefore extending PLH across sensory modalities (Macdonald \& Lavie 2011). In contrast, other studies do not support PLH when examining auditory distractibility (Robinson et al., 2018; Tellinghusien \& Nowak 2003). Robinson et al. (2018) and Tellinghusien and Nowak (2003) examined auditory distractibility while manipulating the perceptual load in the visual modality. These studies found that children (Robinson et al., 2018) and adults (Tellinghusien \& Nowak 2003) were more likely to be slowed down by the auditory distractors in the high load condition, which is inconsistent with PLH. Thus, current research shows unclear answers to the issue of auditory stimuli and PLH, with research both supporting PLH (Macdonald \& Lavie 2011) and research going against PLH (Robinson et al., 2018; Tellinghusien \& Nowak 2003).

Auditory dominance research also predicts that PLH should not predict auditory distractibility (Dunifon, Rivera, \& Robinson, 2016; Robinson \& Sloutsky, 2010). Using variations of change detection tasks (Barnhart, Rivera, \& Robinson, 2018; Dunifon et al., 2016; Robinson, Chandra, \& Sinnett, 2016; Sloutsky \& Napolitano, 2003), participants in these studies had to discriminate auditory and visual information when presented unimodally (just sounds or pictures) or when presented cross-modally (sounds and pictures were paired together). The main finding from these studies is that simultaneously presenting sounds and pictures 
attenuated visual discrimination and/or slowed down visual discrimination compared to the unimodal visual baseline. At the same time, pairing pictures and sounds together often had no negative effect on auditory processing. To account for this finding, Robinson \& Sloutsky (2010) suggested that sensory modalities are competing for attentional resources and that auditory stimuli are favored over visual stimuli. The auditory modality may initially win the competition because auditory stimuli are often transient in nature and it might be adaptive to automatically allocate attention to this information before it disappears (Robinson and Sloutsky, 2010). Thus, based on a proposed mechanism underlying auditory dominance, auditory stimuli should be detected regardless of perceptual load, thus, PLH should not predict which auditory distractors affect processing.

The research presented here followed up on the Robinson et al (2018) study where they examined if PLH could predict auditory and visual distractibility in children, young adults, and older adults. Participants in Robinson et al (2018) were asked to complete four selective attention tasks, which involved detecting a visual target without becoming distracted by auditory and visual distractors under different levels of perceptual load. On each trial, participants were shown a bird or a dog in a box and were asked to press the corresponding button for each target. The visual distractors consisted of a bird or dog (compatible or incompatible with the target in the box) and were presented outside the box. The auditory distractions consisted of a bird chirping or a dog barking (could also be compatible or incompatible with the target). The study found that auditory distractors had a larger effect in young children, whereas, visual distractors had a larger effect in older children, which is consistent with the auditory to visual modality dominance shifts found using other paradigms (Robinson \& Sloutsky, 2004; Sloutsky \& Napolitano, 2003). It was also shown that PLH was unable to predict performance. In contrast to PLH, auditory and visual distractors were more distracting to children under high rather than low load conditions. Finally, there was no evidence that the auditory or visual distractors slowed down adults' responses, however, this may have stemmed from using a task that was geared for young children.

The current study used the same selective attention task from the Robinson et al. (2018) study but we made a few changes to make the task more challenging for adults. First, we shorted the stimuli to $400 \mathrm{~ms}$ (more consistent with previous research in adults), whereas, visual stimuli were presented until participants made a response in Robinson et al (2018). Second, we added a no distractor baseline to assess facilitation and interference effects. If compatible distractors are facilitating processing, then compatible trials should exceed the no distractor baseline, whereas, interference was inferred if performance on incompatible trials was worse than the no distractor baseline.

The primary aim was to test PLH with auditory distractors and to test two hypotheses that may account for the weak auditory interference effects found in Robinson et al (2018). First, it is possible that adults habituated to the auditory stimuli since they were presented on every trial in the auditory distractor conditions. We tested this hypothesis by intermixing auditory and visual distractor trials (intermixed conditions). Thus, on any given trial, participants had no idea if they were going to encounter an auditory distractor, a visual distractor, or no distractor. If habituation to the auditory stimuli can account for weak auditory interference, then the effects of auditory distractors should increase in the current study because the presence of auditory stimuli was less predictable. Second, it is also possible that auditory interference was relatively weak because participants never had to respond to the auditory information, whereas, in most auditory dominance studies participants have to divide attention across sensory modalities and respond to both auditory and visual information (Barnhart et al., 2018; Dunifon et al., 2016; Robinson et al., 2016; Sloutsky \& Napolitano, 2003). To test this hypothesis, we presented an eye or an ear before each trial and this indicated whether the participants should report what they saw or heard (eye/ear conditions). If weak auditory interference stemmed from never having to respond to the auditory stimuli, then distractibility should increase on the eye/ear conditions because participants had to periodically respond to the auditory information. Finally, since we also manipulated perceptual load (low vs. high), the current study will also determine if PLH can predict auditory distractibility. Based on auditory dominance research, it was hypothesized that PLH would not predict performance because auditory stimuli should automatically engage attention and be detected under low and high load.

\section{Methods}

\section{Participants}

Twenty-seven participants $(M=19.41$ years, $S D=.64$ years, 18 Females) from The Ohio State University at Newark participated in the experiment for course credit. One participant was excluded because s/he didn't understand the eye/ear condition. Tests were conducted in a quiet room in the psychology laboratory at The Ohio State University at Newark. Recruitment and experimental procedures were carried out in accordance with the guidelines and approval of The Ohio State University's Behavioral and Social Science Institutional Review Board, Protocol\# 2014B0022. After instructions were given for the study, participants signed and completed an IRB approved informed consent form.

\section{Materials and Design}

The visual stimuli were constructed of six colorful cartoon animals. The stimuli were presented on a 22" Dell $\mathrm{P} 2219 \mathrm{H}$ monitor at approximately $3.80 \mathrm{~cm} \times 5.70 \mathrm{~cm}$, with an approximate horizontal visual angle of $3.63^{\circ}$ and a vertical angle of $5.44^{\circ}$. One of the six visual stimuli presented inside the box was either a Dog or a Bird and was chosen to be the target stimulus. In the low perceptual load condition, only the target stimulus was inside the black-lined box, while the high perceptual load condition had the target stimuli with five 
other non-target animals inside the box (i.e. Frog, Fish, etc.). See Figure 1 for examples of high and low load visual distractor trials. The Bird and Dog targets never appeared inside the box on the same trial, and the exact location of the target and non-targets were shuffled on each trial. The visual stimulus display was presented for $400 \mathrm{~ms}$.

On each trial, the target was either presented with a visual distractor, an auditory distractor, or with no distractor (baseline control). The visual distractors appeared outside the box, within close proximity to the box either above or below. The visual distractors consisted of the Dog or Bird but were outside the box (see Figure 1). Participants were instructed to ignore these visual distractors and report only if they saw the dog or bird inside the box. Half of the visual distractor trials had compatible distractors (the distractor matched the target) and the other half had incompatible distractors (the distractor did not match the target). We also compared performance on these trials to a no distractor baseline. Facilitation was inferred if distractor trials exceeded the no distractor baseline and interference was inferred if distractor trials were worse than the baseline.
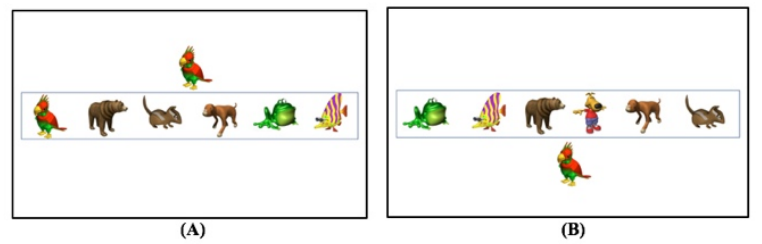

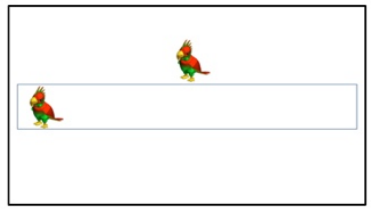

(C)

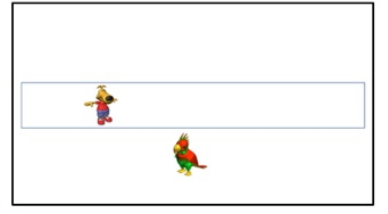

(D)
Figure 1: Examples of the high load (A \& B) and low load (C \& D) visual distractor trials. A \& $\mathrm{C}$ are examples of compatible trials and B \& D are examples of incompatible trials.

The auditory distractors were made up of two distinct sounds. One sound was a bird chirping, while the other was a dog barking. The animal sounds were retrieved from Marcell et al. 2000, yet shortened to approximately $400 \mathrm{~ms}$ using Audacity software, and saved as $44.1 \mathrm{kHz}$.wav files. Auditory stimuli were presented through the use of headphones in the psychology lab. Each sound was approximately $65-68 \mathrm{~dB}$ and was presented at the onset of the visual stimulus. Auditory stimuli were presented to both ears simultaneously with equal intensity. Auditory and visual distractor trials were identical in nature, but only one distractor was presented in a single trial. As with visual distractors, the auditory distractor either matched the visual target on compatible trials (dog paired with dog bark) or it did not match the target on incompatible trials (dog paired with bird chirp). Performance on these trials was compared to the no distractor baseline.

\section{Procedure}

Participants sat approximately $60 \mathrm{~cm}$ from a computer monitor. The experimenter briefly described the experiment and then participants signed the consent form. Once completed, participants filled out a brief demographic form before being presented with the first set of instructions on the computer screen. Participants were shown the small blacklined box in the middle of the screen with either a dog or a bird within the box. Participants were instructed to press the corresponding key on their keyboard when they saw the bird (1 on the numberpad) or a $\operatorname{dog}$ ( 3 on the numberpad). Participants were told that the bird or dog could appear anywhere inside the box and that the bird and dog would never be presented at the same time inside the box. They were also told to respond as quickly and as accurately as possible. Participants were also told that they would only see/hear the stimulus for a half of a second before it disappeared and that the program would not move to the next trial until they responded. Finally, participants were also shown how the computer would try to "trick" them. For example, they were told that the computer may place a bird or dog above or below the box or play the sound of a dog barking or bird chirping through the headphones.

Once the initial instructions were read and the study initiated, the computer randomly started one of the four blocks (high load intermixed, low load intermixed, high load eye/ear, or low load eye/ear). The four blocks were manipulated within-subjects and each block had 120 trials (480 trials in total), with 48 visual distractor trials, 48 auditory distractor trials, and 24 no distractor trials in each block.

In the two intermixed conditions (high and low load), a small fixation point "+" appeared on the screen for $300 \mathrm{~ms}$ prior to each trial. Trial order (visual distractors, auditory distractors, and no distractors) was randomized for each participant. The next trial did not appear until a response was made on the previous trial. In the two eye/ear conditions (high and low load), an image of an eye or an ear appeared in the center of the screen for $300 \mathrm{~ms}$ and this indicated whether the participants should report the auditory distractor or the visual target. In contrast to the intermixed conditions where there were auditory and visual distractor trials, we always presented a visual target with an auditory distractor. On the ear trials, participants reported if the auditory distractor was a bird chirp or a dog bark. These trials were only added to increase attention to the auditory modality. On the eye trials, participants reported if the visual target was a dog or a bird and they heard an auditory distractor in the background. Thus, eye trials were identical to the auditory distractor trials in the intermixed condition, however, participants in this condition had to periodically respond to the auditory distractors. The no distractor baseline trials were identical to the intermixed condition. The whole experiment took approximately $30 \mathrm{~min}$ and response times and accuracies were collected on each trial.

\section{Results}


In both conditions, we examined accuracy (correct or incorrect) and response times (RT). We first focus on the intermixed condition, and then we analyze the eye/ear condition.

\section{Intermixed Conditions}

We calculated two difference scores by subtracting baseline accuracy from compatible and incompatible trials (e.g. compatible difference $=$ compatible accuracy - baseline accuracy). Scores greater than zero showed increased performance compared to baseline and scores less than zero indicated that distractors hurt performance (see Figure 2 for Means and Standard Errors). We submitted accuracy difference scores to a 2 (modality: auditory vs. visual) $\mathrm{x} 2$ (compatibility: compatible vs. incompatible) x 2 (load: high vs. low) repeated measures ANOVA. There was a marginally significant effect of modality, $F(1,26)=3.95, p=.057, \eta_{\mathrm{p}}{ }^{2}$ $=.13$, with visual distractors $(M=-.03, S E=.02)$ being more distracting than auditory distractors $(M=-.01, S E=.01)$. The analysis also revealed an effect of compatibility, $F(1,26)=$ $5.79, p=.023, \eta_{\mathrm{p}}{ }^{2}=.18$, with incompatible distractors $(M=$ $.04, S E=.02)$ decreasing accuracy more than compatible distractors $(M<.01, S E=.01)$. The load x compatibility interaction was not significant, $F(1,26)=1.42, p=.245, \mathrm{\eta}_{\mathrm{p}}^{2}$ $=.05$ ).

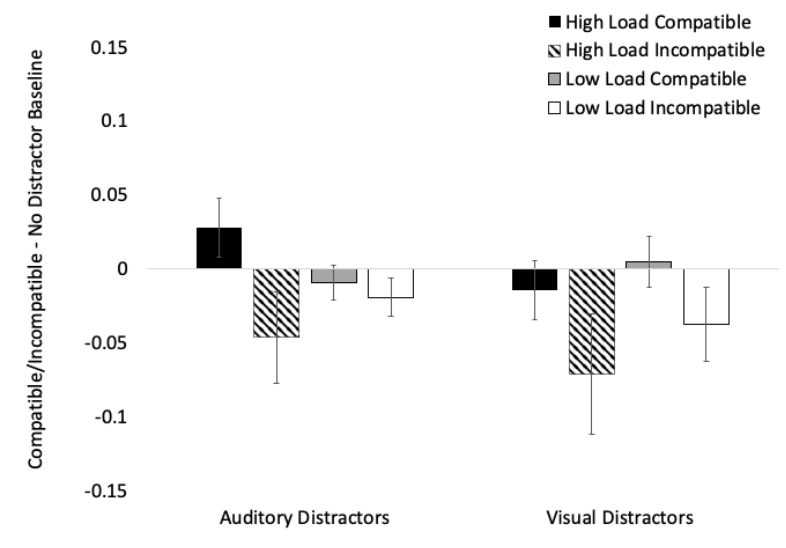

Figure 2: Accuracy difference scores across modality, compatibility, and load in the Intermixed Conditions. Error bars denote Standard Errors.

On each trial we also calculated a response time (timestamp of response - timestamp of stimulus onset), we only analyzed response times on correct trials, and response times greater than two standard deviations were removed. We also calculated difference scores for response times by subtracting baseline response times from compatible and incompatible trials (e.g. compatible RT difference $=$ compatible RT - baseline RT). Scores greater than zero showed slower responses compared to baseline and scores less than zero indicated faster responses compared to baseline (see Figure 3 for Means and Standard Errors).

We submitted RT difference scores to a 2 (modality: auditory vs. visual) x 2 (compatibility: compatible vs. incompatible) x 2 (load: high vs. low) repeated measures ANOVA. There was a significant effect of modality, $F(1,26)$ $=8.23, p=.008, \eta_{\mathrm{p}}{ }^{2}=.24$, with visual distractors $(M=42.61$, $S E=21.29)$ slowing down response times more than auditory distractors $(M=-25.41, S E=16.23)$. The analysis also revealed a marginally significant effect of compatibility, $F(1$, $26)=3.15, p=.087, \eta_{\mathrm{p}}^{2}=.11$, with incompatible distractors $(M=21.85, S E=19.21)$ slowing down responding more than compatible distractors $(M=-4.65, S E=13.30)$. The load by compatibility interaction was not significant, $F(1,26)=.33$, $\left.p=.570, \mathrm{\eta}_{\mathrm{p}}^{2}=.01\right)$.

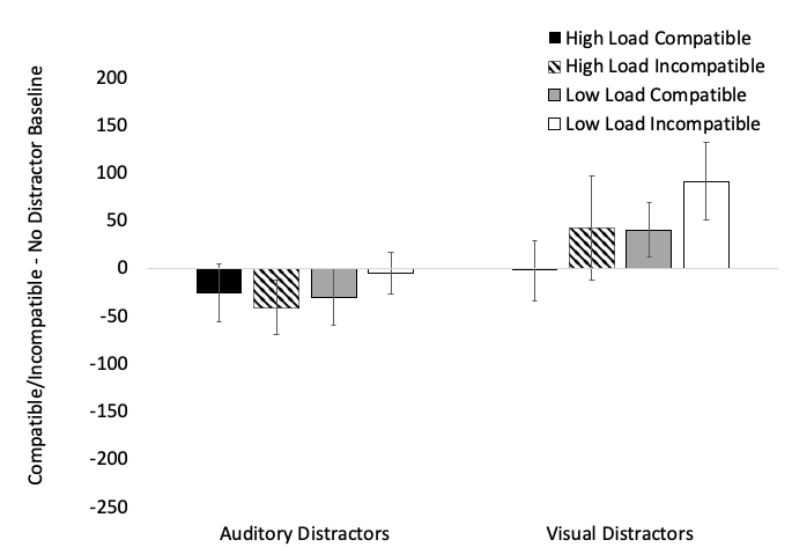

Figure 3: Response Time difference scores across modality, compatibility, and load in the Intermixed Conditions. Error bars denote Standard Errors.

Across accuracy and response time data, visual distractors were more distracting than auditory distractors and incompatible distractors were more distracting than compatible distractors. There was no evidence that auditory distractors facilitated or interfered with responding compared to the no distractor baseline, all one-sample $t$ 's compared to $0, p s>.15$. Finally, the load $\mathrm{x}$ compatibility interaction never reached significance, thus, providing no support for the Perceptual Load Hypothesis.

\section{Eye/Ear Conditions}

As in the Intermixed condition, we calculated accuracy and response time difference scores. Although we had participants respond to the auditory stimuli periodically (ear trials), we were primarily interested in how increased attention to the auditory modality would affect performance while responding to visual targets. Thus, in the analyses below, we only examined accuracy and response times on the visual selective attention task (eye trials). We submitted accuracy difference scores to a 2 (compatibility: compatible vs. incompatible) x 2 (load: high vs. low) repeated measures ANOVA (see Figure 4 for Means and Standard Errors). There was a significant effect of load, $F(1,26)=4.66, p=.040, \mathrm{n}^{2}$ $=.15$, with accuracy being greater under high load $(M<-.01$, $S E=.01)$ than low load $(M=-.05, S E=.02)$. The analysis also revealed an effect of compatibility, $F(1,26)=20.29, p$ 
$\left.<.001, \mathrm{\eta}_{\mathrm{p}}^{2}=.44\right)$, with incompatible distractors $(M=-.09, S E$ $=.02)$ decreasing accuracy compared to compatible distractors $(M=.04, S E=.01)$. The load by compatibility interaction was not significant $F(1,26)=.06, p=.815, \mathrm{\eta}_{\mathrm{p}}{ }^{2}<$ $.01)$.

We also submitted response time difference scores to a 2 (compatibility: compatible vs. incompatible) x 2 (load: high vs. low) repeated measures ANOVA. There was a significant effect of compatibility, $F(1,26)=18.82, p=.987, \eta_{\mathrm{p}}{ }^{2}>.01$, with compatible distractors $(M=-137.99, S E=36.41)$ speeding up responses compared to incompatible distractors $(M=-27.99, S E=39.09)$. The load $\mathrm{x}$ compatibility interaction was significant $F(1,26)=9.04 p=.006, \eta_{\mathrm{p}}^{2}=$ .26), however, the effects were inconsistent with the Perceptual Load Hypothesis. As can be seen in Figure 5, distractor compatibility only had an effect in the high load condition, $t=(26)=4.95, p<.001$, with compatible distractors speeding up responding and no evidence that incompatible auditory distractors significantly slowing down responding (compared to baseline).

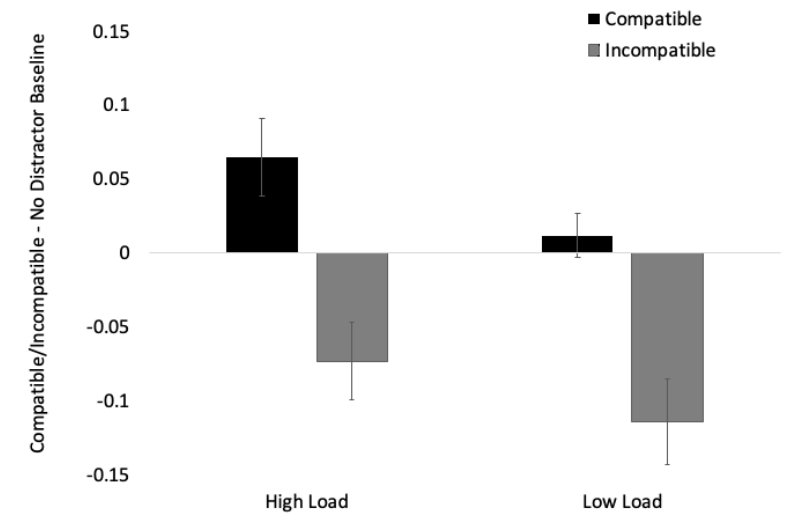

Figure 4: Accuracy difference scores across compatibility and load in the Eye/Ear Conditions. Error bars denote Standard Errors.

In summary, the goal of the eye/ear task was to examine the effects of increased attention to the auditory modality on visual selective attention. The attentional manipulation appeared to have an effect as increased attention to the sounds in the eye/ear task increased the effects of auditory distractor compatibility on the visual selective attention task. For example, in regard to accuracy, auditory distractor compatibility had a larger effect in the eye/ear condition $(d$ $=.87$, Figure 4$)$ than in the intermixed condition $(d=.40$, left side of Figure 2). The same pattern can be seen when examining response times with distractor compatibility having a larger effect in the eye/ear condition $(d=.84$, Figure $5)$ than in the intermixed condition $(d=.06$, left side of Figure 3). While the attention manipulation appeared to increase the effect of distractor compatibility, it is important to note that this effect was either comparable across perceptual load (see accuracy data in Figure 4) or resulted in stronger effects under high load (see Figure 5).

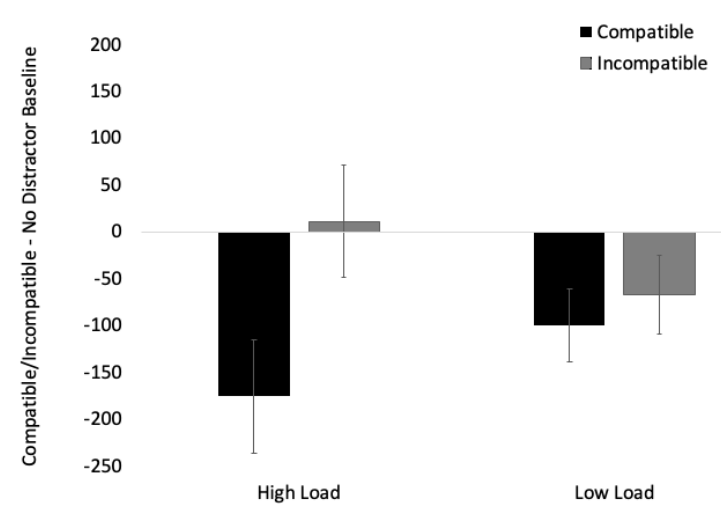

Figure 5: Response time difference scores across compatibility and load in the Eye/Ear Conditions. Error bars denote Standard Errors.

\section{General Discussion}

Participants in the current study completed variations of a visual selective attention task while being presented with auditory and visual distractions under low and high perceptual loads. Auditory and visual distractor trials were either intermixed or were intermixed with an eye or ear presented before each trial, which indicated which stimuli participants were supposed to report. In the intermixed condition, visual distractors were more distracting than auditory distractors, while incompatible distractors decreased performance more than compatible distractors. Data in the intermixed condition provide no support for PLH (Lavie, 1995), however, this likely stemmed from auditory and visual distractors having relatively small effects on the visual responses. Finally, intermixing auditory and visual distractors appeared to have little or no significant effect on auditory distractibility as the effects of compatible and incompatible auditory distractors never differed from the no distractor baselines.

We increased attention to auditory stimuli in the eye/ear condition by requiring participants to periodically respond to the auditory stimuli, and auditory distractor effects were generally stronger in these conditions. For example, as can be seen in Figure 4, compatible distractors increased accuracy and incompatible distractors decreased accuracy; however, the same overall pattern was found across low and high load. The response time analyses showed a slightly different pattern with auditory stimuli speeding up responding in three of the four conditions (see Figure 5). Thus, increasing attention to the auditory modality appeared to speed up responding to the visual targets, as opposed to slowing down responses on incompatible trials. A significant load $\mathrm{x}$ compatibility interaction was found in the eye/ear condition, however, distractor compatibility was more pronounced under high (not low) load.

Research examining the Perceptual Load Hypothesis (PLH) often shows that distractors have a larger effect in low load conditions because of more available resources to detect 
distracting stimuli (Lavie, 1995; Lavie \& Tsal, 1994). While our findings do not support PLH for auditory or visual distractors, the findings are consistent with a growing body of literature showing that compatible and incompatible auditory distractors have a larger effect under high load (Robinson et al., 2018; Tellinghusien \& Nowak, 2003). While future research is needed, it is possible that this effect stemmed from increased cognitive load in the eye/ear task. Under low load, participants with enough cognitive resources may have been able to selectively attend to the visual information and filter auditory distractors. Assuming that the eye/ear task increased cognitive load, participants may have struggled to filter out the distractors because the resources needed to focus attention were depleted due to the complexity of the primary task (see Tellinghusien \& Nowak, 2003, for a similar explanation).

Auditory dominance literature suggests that auditory stimuli should automatically engage attention and disrupt or delay visual processing (see Robinson \& Sloutsky, 2010 for a review). This account predicts that auditory stimuli should have a negative effect on visual selective attention tasks and that PLH should not predict performance because auditory distractors should be detected under low and high load. The current findings are interesting for two reasons. First, auditory stimuli only affected visual processing when participants had to periodically respond to auditory stimuli (eye/ear condition). This has important implications for our understanding of potential mechanisms underlying auditory dominance. While most auditory dominance studies require participants to divide attention across sensory modalities and respond to changes in auditory and visual information (Barnhart et al., 2018; Dunifon et al., 2016; Robinson et al., 2016; Sloutsky \& Napolitano, 2003), participants in the current study only had to respond to a single modality on each trial. This may suggest that auditory stimuli are disrupting response selection, not the encoding of the visual information. Second, while the study provides evidence of facilitation effects, there was little support for auditory dominance/interference. While future research is needed, the facilitation effect likely stems from semantic congruency (Iordanescu, Guzman-Martinez, Grabowecky, \& Suzuki, 2008) with participants responding faster when auditory and visual information provide complementary information (dog paired with dog bark). However, this only provides an explanation for facilitation effects and it is unclear why auditory dominance effects were not found with conflicting auditory information slowing down visual responding. One possibility is that auditory interference occurs after stimulus encoding with effects being most pronounced when sensory modalities are competing for a response.

In summary, selective attention is crucial for many tasks, and it is important to understand what factors account for auditory and visual distractibility. Our findings show that: (a) visual distractors had a larger effect on visual selective attention than auditory distractors and (b) PLH did not predict auditory distractibility, and in fact, we found the opposite effect with distractor compatibility having a larger effect under high perceptual load. This should be further explored in future research, however, this effect likely stems from increased difficulties in filtering distractors under conditions of high cognitive load.

\section{References}

Barnhart, W.R., \& Rivera, S., \& Robinson, C.W. (2018). Different patterns of modality dominance across development. Acta Psychologica, 182, 154-165.

Dunifon, C., Rivera, S., \& Robinson, C.W. (2016). Auditory stimuli automatically grab attention: Evidence from eye tracking and attentional manipulations. Journal of Experimental Psychology: Human Perception and Performance, 42, 1947-1958.

Iordanescu, L., Guzman-Martinez, E., Grabowecky, M., \& Suzuki, S. (2008). Characteristic sounds facilitate visual search. Psychonomic Bulletin and Review, 15, 548-554.

Lavie, N. (1995). Perceptual load as a necessary condition for selective attention. Journal of Experimental Psychology: Human Perception and Performance, 21, 451.

Lavie, N., \& Tsal, Y. (1994). Perceptual load as a major determinant of the locus of selection in visual attention. Attention, Perception, \& Psychophysics, 56, 183-197.

Macdonald, J. S., \& Lavie, N. (2011). Visual perceptual load induces inattentional deafness. Attention, Perception, \& Psychophysics, 73, 1780-1789.

Marcell, M. M., Borella, D., Greene, M., Kerr, E., \& Rogers, S. (2000). Confrontation Naming of Environmental Sounds. Journal of Clinical and Experimental Neuropsychology, 22, 830 - 864.

Murphy, G., Groeger, J. A., \& Greene, C. M. (2016). Twenty years of load theory-Where are we now, and where should we go next? Psychonomic Bulletin \& Review, 23, 1316-1340.

Murphy, S., Spence, C., Dalton, P. (2017). Auditory perceptual load: A review. Hearing Research, 352, 40-48.

Robinson, C.W., Chandra, M., \& Sinnett, S. (2016). Existence of competing modality dominances. Attention, Perception, \& Psychophysics, 78, 1104-1114.

Robinson, C. W., Hawthorn, A. M., \& Rahman, A. N. (2018). Developmental differences in filtering auditory and visual distractors during visual selective attention. Frontiers in Psychology, 9, 1-14.

Robinson, C. W., \& Sloutsky, V. M. (2004). Auditory dominance and its change in the course of development. Child Development, 75, 1387-1401.

Robinson, C. W., \& Sloutsky, V. M. (2010). Development of cross-modal processing. Wiley Interdisciplinary Reviews: Cognitive Science, 1, 135-141.

Sloutsky, V. M., \& Napolitano, A. (2003). Is a picture worth a thousand words? Preference for auditory modality in young children. Child Development, 74, 822-833.

Tellinghuisen, D. J., \& Nowak, E. J. (2003). The inability to ignore auditory distractors as a function of visual task perceptual load. Perception \& Psychophysics, 65(5), 817828. 